\title{
Analytic solutions to nonlinear differential-difference equations by means of the extended $\left(G^{\prime} / G\right)$-expansion method
}

\author{
İsmail Aslan \\ Department of Mathematics, Izmir Institute of Technology, Urla, Izmir 35430, Turkey \\ E-mail: ismailaslan@iyte.edu.tr
}

Received 16 July 2010

Published 31 August 2010

Online at stacks.iop.org/JPhysA/43/395207

\begin{abstract}
In this paper, a discrete extension of the $\left(\mathrm{G}^{\prime} / \mathrm{G}\right)$-expansion method is applied to a relativistic Toda lattice system and a discrete nonlinear Schrödinger equation in order to obtain discrete traveling wave solutions. Closed form solutions with more arbitrary parameters, which reduce to solitary and periodic waves, are exhibited. New rational solutions are also obtained. The method is straightforward and concise, and its applications in physical sciences are promising.
\end{abstract}

PACS numbers: 02.30.Jr, 05.45.Yv, 02.30.Ik

\section{Introduction}

Since the original work of Fermi et al [1] in the 1950s, there has been an explosion of interest to the study of wave phenomena arising in nonlinear differential-difference equations (NDDEs) which are at the very heart of almost any many-particle system. Generally, the dynamics of a many-particle system can be considered as a discrete analog of certain continuous systems. Condensed matter physics is a particular research area of physical sciences where discreteness plays an important role, and the same could also be said of topics in biophysics, chemistry or mechanical engineering. In the last four decades or so, paying more attention to such equations, researchers proposed many physically important NDDEs [2-5].

Recently, Wang et al [6] proposed the so-called $\left(\mathrm{G}^{\prime} / \mathrm{G}\right)$-expansion method to seek for exact solutions of nonlinear evolution equations in the form of solitary and periodic waves. The essential observation about the $\left(\mathrm{G}^{\prime} / \mathrm{G}\right)$-expansion method is that it reveals further results with more arbitrary parameters and is powerful in the sense that it takes full advantage of linear theory by assuming a second-order linear equation as the ansatz. The solution procedure is easy, reliable and efficient, as well as does not require a large amount of run-time with the help of a computer algebra system such as MATHEMATICA. Naturally, the $\left(G^{\prime} / G\right)$-expansion 
method has been applied to various kinds of nonlinear problems in science and engineering, and lately more attention is paid to its adaptation and generalization [7-23].

Our objective in this paper is to perform an analytic study on a relativistic Toda lattice system and a discrete nonlinear Schrödinger equation by using the extended $\left(G^{\prime} / G\right)$-expansion method. The rest of this paper is organized as follows. The method of solution is described in section 2. We analyze two physically important equations in section 3. Finally, some concluding remarks are given in section 4.

\section{The extended $\left(\mathbf{G}^{\prime} / \mathbf{G}\right)$-expansion method for NDDEs}

Let us consider a system of $M$ polynomial NDDEs in the form

$\Delta\left(\mathbf{u}_{\mathbf{n}+\mathbf{p}_{1}}(\mathbf{x}), \ldots, \mathbf{u}_{\mathbf{n}+\mathbf{p}_{k}}(\mathbf{x}), \ldots, \mathbf{u}_{\mathbf{n}+\mathbf{p}_{1}}^{\prime}(\mathbf{x}), \ldots, \mathbf{u}_{\mathbf{n}+\mathbf{p}_{k}}^{\prime}(\mathbf{x}), \ldots, \mathbf{u}_{\mathbf{n}+\mathbf{p}_{1}}^{(r)}(\mathbf{x}), \ldots, \mathbf{u}_{\mathbf{n}+\mathbf{p}_{k}}^{(r)}(\mathbf{x})\right)=0$,

in which the dependent variable $\mathbf{u}_{\mathbf{n}}$ have $M$ components $u_{i, \mathrm{n}}$ and so do its shifts, the continuous variable $\mathbf{x}$ has $N$ components $x_{i}$, the discrete variable $\mathbf{n}$ has $Q$ components $n_{j}$, and the $k$ shift vectors $\mathbf{p}_{i} \mathbb{Z}^{Q}$ and $\mathbf{u}^{(r)}(\mathbf{x})$ denote the collection of mixed derivative terms of order $r$.

Step 1. For traveling wave solutions to equation (1), we first make the wave transformation

$\mathbf{u}_{\mathbf{n}+\mathbf{p}_{s}}(\mathbf{x})=\mathbf{U}_{\mathbf{n}+\mathbf{p}_{s}}\left(\xi_{\mathbf{n}}\right), \xi_{\mathbf{n}}=\sum_{i=1}^{Q} d_{i} n_{i}+\sum_{j=1}^{N} c_{j} x_{j}+\zeta \quad(s=1,2, \ldots, k)$,

where the coefficients $c_{1}, c_{2}, \ldots, c_{N}, d_{1}, d_{2}, \ldots, d_{Q}$ and the phase $\zeta$ are all constants. Then, equation (1) reduces to

$$
\begin{aligned}
\Delta\left(\mathbf{U}_{\mathbf{n}+\mathbf{p}_{1}}\left(\xi_{\mathbf{n}}\right), \ldots, \mathbf{U}_{\mathbf{n}+\mathbf{p}_{k}}\left(\xi_{\mathbf{n}}\right), \ldots, \mathbf{U}_{\mathbf{n}+\mathbf{p}_{1}}^{\prime}\left(\xi_{\mathbf{n}}\right), \ldots, \mathbf{U}_{\mathbf{n}+\mathbf{p}_{k}}^{\prime}\left(\xi_{\mathbf{n}}\right), \ldots, \mathbf{U}_{\mathbf{n}+\mathbf{p}_{1}}^{(r)}\left(\xi_{\mathbf{n}}\right), \ldots,\right. \\
\left.\mathbf{U}_{\mathbf{n}+\mathbf{p}_{k}}^{(r)}\left(\xi_{\mathbf{n}}\right)\right)=0 .
\end{aligned}
$$

Step 2. We assume that the solution of equation (3) is in the finite series expansion form

$$
\mathbf{U}_{\mathbf{n}}\left(\xi_{\mathbf{n}}\right)=\sum_{l=-m}^{m} a_{l}\left(\frac{G^{\prime}\left(\xi_{\mathbf{n}}\right)}{G\left(\xi_{\mathbf{n}}\right)}\right)^{l}, \quad a_{-m}^{2}+a_{m}^{2} \neq 0,
$$

where $m$ (a positive integer) and $a_{i}$ 's are constants to be determined, and $G\left(\xi_{\mathbf{n}}\right)$ is the general solution of the equation

$$
G^{\prime \prime}\left(\xi_{\mathbf{n}}\right)+\mu G\left(\xi_{\mathbf{n}}\right)=0
$$

where $\mu$ is an arbitrary constant and the prime denotes the derivative with respect to $\xi_{\mathbf{n}}$. The general solution of equation (5) is well known for us. Thus, we have the following cases:

$$
\begin{aligned}
& \frac{G^{\prime}\left(\xi_{\mathbf{n}}\right)}{G\left(\xi_{\mathbf{n}}\right)}=\sqrt{-\mu}\left(\frac{C_{1} \cosh \left(\sqrt{-\mu} \xi_{\mathbf{n}}\right)+C_{2} \sinh \left(\sqrt{-\mu} \xi_{\mathbf{n}}\right)}{C_{1} \sinh \left(\sqrt{-\mu} \xi_{\mathbf{n}}\right)+C_{2} \cosh \left(\sqrt{-\mu} \xi_{\mathbf{n}}\right)}\right), \quad \mu<0, \\
& \frac{G^{\prime}\left(\xi_{\mathbf{n}}\right)}{G\left(\xi_{\mathbf{n}}\right)}=\sqrt{\mu}\left(\frac{-C_{1} \sin \left(\sqrt{\mu} \xi_{\mathbf{n}}\right)+C_{2} \cos \left(\sqrt{\mu} \xi_{\mathbf{n}}\right)}{C_{1} \cos \left(\sqrt{\mu} \xi_{\mathbf{n}}\right)+C_{2} \sin \left(\sqrt{\mu} \xi_{\mathbf{n}}\right)}\right), \quad \mu>0, \\
& \frac{G^{\prime}\left(\xi_{\mathbf{n}}\right)}{G\left(\xi_{\mathbf{n}}\right)}=\frac{C_{1}}{C_{1} \xi_{\mathbf{n}}+C_{2}}, \quad \mu=0,
\end{aligned}
$$

where $C_{1}$ and $C_{2}$ are arbitrary constants.

Step 3. By a straightforward calculation, we can get the identity

$$
\xi_{\mathbf{n}+\mathbf{p}_{s}}=\xi_{\mathbf{n}}+\varphi_{s}, \quad \varphi_{s}=p_{s 1} d_{1}+p_{s 2} d_{2}+\cdots+p_{s} d_{Q},
$$


where $p_{s j}$ is the $j$ th component of the shift vector $\mathbf{p}_{s}$. Hence, considering the trigonometric/hyperbolic function identities and using the functions $(6 a)-(6 c)$ together with (7), we derive the uniform formulas:

$\mathbf{U}_{\mathbf{n}+\mathbf{p}_{s}}\left(\xi_{\mathbf{n}}\right)=\sum_{l=-m}^{m} a_{l}\left(\frac{\sqrt{-\mu} G^{\prime}\left(\xi_{\mathbf{n}}\right)-\mu \tanh \left(\sqrt{-\mu} \varphi_{s}\right) G\left(\xi_{\mathbf{n}}\right)}{\sqrt{-\mu} G\left(\xi_{\mathbf{n}}\right)+\tanh \left(\sqrt{-\mu} \varphi_{s}\right) G^{\prime}\left(\xi_{\mathbf{n}}\right)}\right)^{l}, \quad a_{-m}^{2}+a_{m}^{2} \neq 0, \quad \mu<0$,

$\mathbf{U}_{\mathbf{n}+\mathbf{p}_{s}}\left(\xi_{\mathbf{n}}\right)=\sum_{l=-m}^{m} a_{l}\left(\frac{\sqrt{\mu} G^{\prime}\left(\xi_{\mathbf{n}}\right)-\mu \tan \left(\sqrt{\mu} \varphi_{s}\right) G\left(\xi_{\mathbf{n}}\right)}{\sqrt{\mu} G\left(\xi_{\mathbf{n}}\right)+\tan \left(\sqrt{\mu} \varphi_{s}\right) G^{\prime}\left(\xi_{\mathbf{n}}\right)}\right)^{l}, \quad a_{-m}^{2}+a_{m}^{2} \neq 0, \quad \mu>0$,

$\mathbf{U}_{\mathbf{n}+\mathbf{p}_{s}}\left(\xi_{\mathbf{n}}\right)=\sum_{l=-m}^{m} a_{l}\left(\frac{G^{\prime}\left(\xi_{\mathbf{n}}\right)}{G\left(\xi_{\mathbf{n}}\right)+\varphi_{s} G^{\prime}\left(\xi_{\mathbf{n}}\right)}\right)^{l}, \quad a_{-m}^{2}+a_{m}^{2} \neq 0, \quad \mu=0$.

Step 4. By means of the ansatz (4), we define the degree of $\mathbf{U}_{\mathbf{n}}\left(\xi_{\mathbf{n}}\right)$ as $D\left[\mathbf{U}_{\mathbf{n}}\left(\xi_{\mathbf{n}}\right)\right]=m$ which gives rise to the degree of other expressions as

$$
\begin{aligned}
& D\left[\mathbf{U}_{\mathbf{n}}^{(r)}\left(\xi_{\mathbf{n}}\right)\right]=m+r, \quad D\left[\left(\mathbf{U}_{\mathbf{n}}^{(r)}\left(\xi_{\mathbf{n}}\right)\right)^{\beta}\right]=\beta(m+r), \\
& D\left[\left(\mathbf{U}_{\mathbf{n}}\left(\xi_{\mathbf{n}}\right)\right)^{\alpha}\left(\mathbf{U}_{\mathbf{n}}^{(r)}\left(\xi_{\mathbf{n}}\right)\right)^{\beta}\right]=\alpha m+\beta(m+r)
\end{aligned}
$$

Balancing the highest order nonlinear term(s) and the highest order derivative term in $\mathbf{U}_{\mathbf{n}}\left(\xi_{\mathbf{n}}\right)$, we can easily determine the degree $m$ of equations (4) and $(8 a)-(8 c)$ from equation (3). Since $\mathbf{U}_{\mathbf{n}+\mathbf{p}_{s}}$ can be interpreted as being of degree zero in $\left(G^{\prime}\left(\xi_{\mathbf{n}}\right) / G\left(\xi_{\mathbf{n}}\right)\right)$, the leading terms of $\mathbf{U}_{\mathbf{n}+\mathbf{p}_{s}}\left(\mathbf{p}_{s} \neq 0\right)$ will not have any affect on the balancing procedure.

Step 5. Substituting the ansätze (4) and (8a)-(8c) together with (5) into equation (3), equating the coefficients of $\left(G^{\prime}\left(\xi_{\mathbf{n}}\right) / G\left(\xi_{\mathbf{n}}\right)\right)^{l}(l=0,1,2, \ldots)$ to zero, we obtain a system of nonlinear algebraic equations from which the undetermined constants $a_{l}, d_{i}, c_{j}$ and $\mu$ can be explicitly found. Substituting these results into (4), we can derive various kinds of discrete exact solutions to equation (1).

Note 1. It is worth to mention that there are three improved computational steps in our algorithm to obtain more wider results in a concise manner. First, the extended method leads to the solution of the form (4) in which the sum goes from $l=-m$ to $l=m$ instead of from $l=0$ to $l=m$. Second, the standard method $[6,20]$ uses the auxiliary equation $G^{\prime \prime}+\lambda G^{\prime}+\mu G=0$ as the ansatz. Without loss of generality, we consider the auxiliary equation (5) by taking $\lambda=0$. This approach provides equivalent results with the original method. However, it is more advantageous since it minimizes the number of parameters, see [24]. Third, contrary to the procedure [20], we consider another case (namely, the case (8c)) for the inclusion of rational solutions.

\section{Applications}

In this section, we apply the algorithm described in the preceding section to some NDDEs. 


\subsection{Relativistic Toda lattice system}

One of the most famous models for discrete solitons is the integrable Toda lattice system [25]:

$$
\left\{\begin{array}{l}
\frac{\mathrm{d} u_{n}}{\mathrm{~d} t}=\left(u_{n+1}-v_{n}\right) v_{n}-\left(u_{n-1}-v_{n-1}\right) v_{n-1}, \\
\frac{\mathrm{d} v_{n}}{\mathrm{~d} t}=v_{n}\left(u_{n+1}-u_{n}\right) .
\end{array}\right.
$$

For solving equation (9), we first let

$$
u_{n}=U_{n}\left(\xi_{n}\right), \quad v_{n}=V_{n}\left(\xi_{n}\right), \quad \xi_{n}=\mathrm{d} n+c t+\zeta,
$$

where $c$ and $d$ are constants to be determined and $\zeta$ denotes the phase shift. Then, equation (9) can be reduced to

$$
\left\{\begin{array}{l}
c U_{n}^{\prime}-\left(U_{n+1}-V_{n}\right) V_{n}+\left(U_{n-1}-V_{n-1}\right) V_{n-1}=0, \\
c V_{n}^{\prime}-V_{n}\left(U_{n+1}-U_{n}\right)=0,
\end{array}\right.
$$

where $U_{n}=U_{n}\left(\xi_{n}\right), V_{n}=V_{n}\left(\xi_{n}\right)$ and the prime denotes the derivative with respect to $\xi_{n}$. We expand the solution of (11) in the frame (4). Balancing the linear term of the highest order with the highest nonlinear term in (11) yields to $m=1$. Thus, we look for solutions of equation (11) in the form

$$
\begin{cases}U_{n}=a_{0}+a_{1}\left(\frac{G^{\prime}}{G}\right)+a_{-1}\left(\frac{G^{\prime}}{G}\right)^{-1}, & a_{-1}^{2}+a_{1}^{2} \neq 0, \\ V_{n}=b_{0}+b_{1}\left(\frac{G^{\prime}}{G}\right)+b_{-1}\left(\frac{G^{\prime}}{G}\right)^{-1}, & b_{-1}^{2}+b_{1}^{2} \neq 0,\end{cases}
$$

where $G=G\left(\xi_{n}\right)$ satisfies equation (5), and $a_{0}, a_{1}, a_{-1}, b_{0}, b_{1}, b_{-1}$ are arbitrary constants to be specified.

Case 1. When $\mu<0$, from (8a), we have

$$
\begin{aligned}
& U_{n \pm 1}=\sum_{l=-1}^{1} a_{l}\left(\frac{\sqrt{-\mu} G^{\prime} \mp \mu \tanh \left(\sqrt{-\mu} \varphi_{s}\right) G}{\sqrt{-\mu} G \pm \tanh \left(\sqrt{-\mu} \varphi_{s}\right) G^{\prime}}\right)^{l} \\
& V_{n \pm 1}=\sum_{l=-1}^{1} b_{l}\left(\frac{\sqrt{-\mu} G^{\prime} \mp \mu \tanh \left(\sqrt{-\mu} \varphi_{s}\right) G}{\sqrt{-\mu} G \pm \tanh \left(\sqrt{-\mu} \varphi_{s}\right) G^{\prime}}\right)^{l} .
\end{aligned}
$$

Substituting the ansatz (12) and the expressions (13a) and (13b) along with equation (5) into equation (11), clearing the denominator and equating the coefficients of $\left(G^{\prime} / G\right)^{l}(0 \leqslant l \leqslant 10)$ to zero, we obtain a system of nonlinear algebraic equations for $a_{0}, a_{1}, a_{-1}, b_{0}, b_{1}, b_{-1}, c, d$ and $\mu$. Solving the system (we will omit to display them for simplicity) simultaneously, we get the following solution sets:

$\left\{a_{0}=c \sqrt{-\mu} \operatorname{coth}(d \sqrt{-\mu}), b_{0}=c \sqrt{-\mu} \operatorname{coth}(d \sqrt{-\mu}), a_{-1}=0, a_{1}=c, b_{-1}=0, b_{1}=c\right\}$,

$\left\{a_{0}=2 c \sqrt{-\mu} \operatorname{coth}(2 d \sqrt{-\mu}), b_{0}=2 c \sqrt{-\mu} \operatorname{coth}(2 d \sqrt{-\mu})\right.$,

$$
\left.a_{-1}=-c \mu, a_{1}=c, b_{-1}=-c \mu, b_{1}=c\right\}
$$

and the corresponding discrete hyperbolic function solutions to equation (9) as

$$
\left\{\begin{array}{l}
u_{n, 1}(t)=c \sqrt{-\mu}\left(w_{n}(t)+\operatorname{coth}(d \sqrt{-\mu})\right), \\
v_{n, 1}(t)=c \sqrt{-\mu}\left(w_{n}(t)+\operatorname{coth}(d \sqrt{-\mu})\right)
\end{array}\right.
$$




$$
\left\{\begin{array}{l}
u_{n, 2}(t)=c \sqrt{-\mu}\left(\frac{1}{w_{n}(t)}+w_{n}(t)+2 \operatorname{coth}(2 d \sqrt{-\mu})\right) \\
v_{n, 2}(t)=c \sqrt{-\mu}\left(\frac{1}{w_{n}(t)}+w_{n}(t)+2 \operatorname{coth}(2 d \sqrt{-\mu})\right)
\end{array}\right.
$$

where

$$
w_{n}(t)=\frac{C_{1} \cosh (\sqrt{-\mu}(\mathrm{d} n+c t+\zeta))+C_{2} \sinh (\sqrt{-\mu}(\mathrm{d} n+c t+\zeta))}{C_{1} \sinh (\sqrt{-\mu}(\mathrm{d} n+c t+\zeta))+C_{2} \cosh (\sqrt{-\mu}(\mathrm{d} n+c t+\zeta))},
$$

in which $\mu<0, d, c, \zeta, C_{1}, C_{2}$ are arbitrary constants.

As a special example, if we let ' $C_{1}=0$ and $C_{2} \neq 0$ ' or ' $C_{1} \neq 0$ and $C_{2}=0$ ' in (15a) respectively, then we get formal discrete solitary wave solutions to equation (9) as follows:

$$
\begin{array}{r}
\left\{\begin{array}{l}
u_{n, 3}(t)=c \sqrt{-\mu}(\tanh (\sqrt{-\mu}(\mathrm{d} n+c t+\zeta))+\operatorname{coth}(d \sqrt{-\mu})), \\
v_{n, 3}(t)=c \sqrt{-\mu}(\tanh (\sqrt{-\mu}(\mathrm{d} n+c t+\zeta))+\operatorname{coth}(d \sqrt{-\mu})),
\end{array}\right. \\
\left\{\begin{array}{l}
u_{n, 4}(t)=c \sqrt{-\mu}(\operatorname{coth}(\sqrt{-\mu}(\mathrm{d} n+c t+\zeta))+\operatorname{coth}(d \sqrt{-\mu})), \\
v_{n, 4}(t)=c \sqrt{-\mu}(\operatorname{coth}(\sqrt{-\mu}(\mathrm{d} n+c t+\zeta))+\operatorname{coth}(d \sqrt{-\mu})),
\end{array}\right.
\end{array}
$$

where $\mu<0, d, c$ and $\zeta$ are arbitrary constants.

Case 2. When $\mu>0$, from (8b), we have

$$
\begin{aligned}
& U_{n \pm 1}=\sum_{l=-1}^{1} a_{l}\left(\frac{\sqrt{\mu} G^{\prime} \mp \mu \tan \left(\sqrt{\mu} \varphi_{s}\right) G}{\sqrt{\mu} G \pm \tan \left(\sqrt{\mu} \varphi_{s}\right) G^{\prime}}\right)^{l}, \\
& V_{n \pm 1}=\sum_{l=-1}^{1} b_{l}\left(\frac{\sqrt{\mu} G^{\prime} \mp \mu \tan \left(\sqrt{\mu} \varphi_{s}\right) G}{\sqrt{\mu} G \pm \tan \left(\sqrt{\mu} \varphi_{s}\right) G^{\prime}}\right)^{l} .
\end{aligned}
$$

Substituting the ansatz (12) and the expressions (19a), (19b) along with equation (5) into equation (11), clearing the denominator and equating the coefficients of $\left(G^{\prime} / G\right)^{l}(0 \leqslant l \leqslant 10)$ to zero, we obtain a system of nonlinear algebraic equations for $a_{0}, a_{1}, a_{-1}, b_{0}, b_{1}, b_{-1}, c, d$ and $\mu$. Solving the system simultaneously, we get the following solution sets:

$\left\{a_{0}=c \sqrt{\mu} \cot (d \sqrt{\mu}), b_{0}=c \sqrt{\mu} \cot (d \sqrt{\mu}), a_{-1}=0, a_{1}=c, b_{-1}=0, b_{1}=c\right\}$

$\left\{a_{0}=2 c \sqrt{\mu} \cot (2 d \sqrt{\mu}), b_{0}=2 c \sqrt{\mu} \cot (2 d \sqrt{\mu}), a_{-1}=-c \mu, a_{1}=c, b_{-1}=-c \mu, b_{1}=c\right\}$,

and the corresponding discrete trigonometric function solutions to equation (9) as

$$
\begin{aligned}
& \left\{\begin{array}{l}
u_{n, 5}(t)=c \sqrt{\mu}\left(w_{n}(t)+\cot (d \sqrt{\mu})\right), \\
v_{n, 5}(t)=c \sqrt{\mu}\left(w_{n}(t)+\cot (d \sqrt{\mu})\right),
\end{array}\right. \\
& \left\{\begin{array}{l}
u_{n, 6}(t)=c \sqrt{\mu}\left(-\frac{1}{w_{n}(t)}+w_{n}(t)+2 \cot (2 d \sqrt{\mu})\right), \\
v_{n, 6}(t)=c \sqrt{\mu}\left(-\frac{1}{w_{n}(t)}+w_{n}(t)+2 \cot (2 d \sqrt{\mu})\right),
\end{array}\right.
\end{aligned}
$$

where

$$
w_{n}(t)=\frac{C_{2} \cos (\sqrt{\mu}(\mathrm{d} n+c t+\zeta))-C_{1} \sin (\sqrt{\mu}(\mathrm{d} n+c t+\zeta))}{C_{1} \cos (\sqrt{\mu}(\mathrm{d} n+c t+\zeta))+C_{2} \sin (\sqrt{\mu}(\mathrm{d} n+c t+\zeta))}
$$


in which $\mu>0, d, c, \zeta, C_{1}$ and $C_{2}$ are arbitrary constants.

As a special example, if we take ' $C_{1}=0$ and $C_{2} \neq 0$ ' or ' $C_{1} \neq 0$ and $C_{2}=0$ ' in the expression $(21 a)$ respectively, then we get formal discrete periodic wave solutions to equation (9) as follows:

$$
\begin{gathered}
\left\{\begin{array}{l}
u_{n, 7}(t)=c \sqrt{\mu}(\cot (\sqrt{\mu}(\mathrm{d} n+c t+\zeta))+\cot (d \sqrt{\mu})), \\
v_{n, 7}(t)=c \sqrt{\mu}(\cot (\sqrt{\mu}(\mathrm{d} n+c t+\zeta))+\cot (d \sqrt{\mu})),
\end{array}\right. \\
\left\{\begin{array}{l}
u_{n, 8}(t)=c \sqrt{\mu}(-\tan (\sqrt{\mu}(\mathrm{d} n+c t+\zeta))+\cot (d \sqrt{\mu})), \\
v_{n, 8}(t)=c \sqrt{\mu}(-\tan (\sqrt{\mu}(\mathrm{d} n+c t+\zeta))+\cot (d \sqrt{\mu})),
\end{array}\right.
\end{gathered}
$$

where $\mu>0, d, c$ and $\zeta$ are arbitrary constants.

Case 3. When $\mu=0$, from (8c), we have

$$
\begin{aligned}
U_{n \pm 1} & =\sum_{l=-1}^{1} a_{l}\left(\frac{G^{\prime}}{G \pm \varphi_{s} G^{\prime}}\right)^{l} \\
V_{n \pm 1} & =\sum_{l=-1}^{1} b_{l}\left(\frac{G^{\prime}}{G \pm \varphi_{s} G^{\prime}}\right)^{l} .
\end{aligned}
$$

Substituting the ansatz (12) and the expressions (25a) and (25b) along with equation (5) into equation (11), clearing the denominator and equating the coefficients of $\left(G^{\prime} / G\right)^{l}(0 \leqslant l \leqslant 10)$ to zero, we obtain a system of nonlinear algebraic equations for $a_{0}$, $a_{1}, a_{-1}, b_{0}, b_{1}, b_{-1}, c$ and $d$. Solving the system simultaneously, we get the following solution set:

$$
\left\{a_{0}=\frac{c}{d}, a_{1}=c, b_{1}=c, b_{0}=\frac{c}{d}, a_{-1}=0, b_{-1}=0\right\}
$$

and the corresponding discrete rational function solution to equation (9) as

$$
\left\{\begin{array}{l}
u_{n, 9}(t)=c\left(\frac{1}{d}+\frac{C_{1}}{C_{1}(\mathrm{~d} n+c t+\zeta)+C_{2}}\right), \\
v_{n, 9}(t)=c\left(\frac{1}{d}+\frac{C_{1}}{C_{1}(\mathrm{~d} n+c t+\zeta)+C_{2}}\right),
\end{array}\right.
$$

where $c, d, \zeta, C_{1}$ and $C_{2}$ are arbitrary constants.

Note 2. We observe that our solution (17) coincides with the solution of Baldwin et al [25] while our other solutions do not appear there. To the best of our knowledge, our rational solution (27) is presented here for the first time.

\subsection{The discrete nonlinear Schrödinger equation}

We now consider the integrable discrete nonlinear Schrödinger equation [26]

$\mathrm{i} \frac{\mathrm{d} u_{n}(t)}{\mathrm{d} t}+\alpha\left(u_{n+1}(t)-2 u_{n}(t)+u_{n-1}(t)\right)+\beta\left|u_{n}(t)\right|^{2}\left(u_{n+1}(t)+u_{n-1}(t)\right)=0$,

where $u_{n}(t)=u(n, t)$ denotes the displacement of the $n$th particle from the equilibrium position, $i=\sqrt{-1}$, and $\alpha$, and $\beta$ are nonzero real constants.

For solving equation (28), we first make the traveling wave transformation

$$
u_{n}=\mathrm{e}^{\mathrm{i} \theta_{n}} \phi_{n}\left(\xi_{n}\right), \quad \theta_{n}=d_{1} n+c_{1} t+\zeta_{1}, \quad \xi_{n}=d_{2} n+c_{2} t+\zeta_{2},
$$

6 
and

$$
u_{n+1}=\mathrm{e}^{\mathrm{i} \theta_{n}} \mathrm{e}^{\mathrm{i} d_{1}} \phi_{n+1}\left(\xi_{n}\right), \quad u_{n-1}=\mathrm{e}^{\mathrm{i} \theta_{n}} \mathrm{e}^{-\mathrm{i} d_{1}} \phi_{n-1}\left(\xi_{n}\right),
$$

where $\phi_{n}=\phi_{n}\left(\xi_{n}\right)$ is a real-valued function, $d_{1}$ and $c_{1}$ are the wave number of the carrier wave and the frequency, $c_{2}$ and $d_{2}$ are related to the group velocity and the pulse width, $\zeta_{1}$ and $\zeta_{2}$ denote the initial phases. Now, using the Euler formula $\mathrm{e}^{ \pm \mathrm{i} d_{1}}=\cos d_{1} \pm \mathrm{i} \sin d_{1}$, equation (28) can be reduced to the system

$$
\left\{\begin{array}{l}
-c_{1} \phi_{n}+\cos \left(d_{1}\right)\left(\alpha+\beta \phi_{n}^{2}\right)\left(\phi_{n+1}+\phi_{n-1}\right)-2 \alpha \phi_{n}=0 \\
c_{2} \phi_{n}^{\prime}+\sin \left(d_{1}\right)\left(\alpha+\beta \phi_{n}^{2}\right)\left(\phi_{n+1}-\phi_{n-1}\right)=0
\end{array}\right.
$$

where the prime denotes the derivative with respect to $\xi_{n}$. We expand the solution of (31) in the form of (4). Balancing the linear term of the highest order with the highest nonlinear term in (31) leads to $m=1$. Thus, for the traveling wave solutions of (31), we assume the ansatz

$$
\phi_{n}=a_{0}+a_{1}\left(\frac{G^{\prime}}{G}\right)+a_{-1}\left(\frac{G^{\prime}}{G}\right)^{-1}, \quad a_{-1}^{2}+a_{1}^{2} \neq 0
$$

where $G=G\left(\xi_{n}\right)$ satisfies equation (5), and $a_{0}, a_{1}$, and $a_{-1}$ are arbitrary constants to be determined. Because the procedure is similar to the scheme used in section 3.1, we will omit most of the details here.

Case 1. $\mu<0$.

In this case, we first derive the expressions $\phi_{n \pm 1}$ in accordance with $(8 a)$ and substitute them along with (32) into equation (31). Then, clearing the denominator and setting the coefficients of $\left(G^{\prime} / G\right)^{l}(0 \leqslant l \leqslant 8)$ to zero, we derive a system of nonlinear algebraic equations for $a_{0}, a_{1}, a_{-1}, d_{1}, d_{2}, c_{1}, c_{2}$ and $\mu$. Solving the system, we get the following solution set:

$$
\left\{\begin{aligned}
& c_{1}= 2 \alpha\left(-1+\cos \left(d_{1}\right) \operatorname{sech}^{2}\left(\sqrt{-\mu} d_{2}\right)\right), \quad c_{2}=-2 \alpha \sin \left(d_{1}\right) \tanh \left(\sqrt{-\mu} d_{2}\right) / \sqrt{-\mu}, \\
& a_{0}=0, \quad a_{-1}=0 \\
& a_{1}=\mp \sqrt{-\alpha / \beta} \tanh \left(\sqrt{-\mu} d_{2}\right) / \sqrt{-\mu}
\end{aligned}\right\}
$$

and the corresponding discrete hyperbolic function solution to equation (28) as

$$
\begin{aligned}
u_{n, 1}^{\mp}(t)=\mp & \frac{\sqrt{-\alpha} \tanh \left(\sqrt{-\mu} d_{2}\right)}{\sqrt{\beta}}\left(\frac{C_{1} \cosh \left(\sqrt{-\mu} \xi_{n}\right)+C_{2} \sinh \left(\sqrt{-\mu} \xi_{n}\right)}{C_{1} \sinh \left(\sqrt{-\mu} \xi_{n}\right)+C_{2} \cosh \left(\sqrt{-\mu} \xi_{n}\right)}\right) \\
& \times \exp \left(\mathrm{i}\left(d_{1} n+2 \alpha\left(-1+\cos \left(d_{1}\right) \operatorname{sech}^{2}\left(\sqrt{-\mu} d_{2}\right)\right) t+\zeta_{1}\right)\right), \quad \frac{\alpha}{\beta}<0,
\end{aligned}
$$

where $\xi_{n}=d_{2} n-\frac{2 \alpha \sin \left(d_{1}\right) \tanh \left(\sqrt{-\mu} d_{2}\right)}{\sqrt{-\mu}} t+\zeta_{2}$, and $\mu<0, d_{1}, d_{2}, \zeta_{1}, \zeta_{2}, C_{1}$ and $C_{2}$ remain arbitrary.

As a particular example, if we let ' $C_{1}=0$ and $C_{2} \neq 0$ ' or ' $C_{1} \neq 0$ and $C_{2}=0$ ' in (34) respectively, then we get formal discrete solitary wave solutions to equation (28) as follows:

$$
\begin{aligned}
u_{n, 2}^{\mp}(t)=\mp & \frac{\sqrt{-\alpha} \tanh \left(\sqrt{-\mu} d_{2}\right)}{\sqrt{\beta}} \tanh \left(\sqrt{-\mu}\left(d_{2} n-\frac{2 \alpha \sin \left(d_{1}\right) \tanh \left(\sqrt{-\mu} d_{2}\right)}{\sqrt{-\mu}} t+\zeta_{2}\right)\right) \\
& \times \exp \left(\mathrm{i}\left(d_{1} n+2 \alpha\left(-1+\cos \left(d_{1}\right) \operatorname{sech}^{2}\left(\sqrt{-\mu} d_{2}\right)\right) t+\zeta_{1}\right)\right), \quad \frac{\alpha}{\beta}<0, \\
u_{n, 3}^{\mp}(t)=\mp & \frac{\sqrt{-\alpha} \tanh \left(\sqrt{-\mu} d_{2}\right)}{\sqrt{\beta}} \operatorname{coth}\left(\sqrt{-\mu}\left(d_{2} n-\frac{2 \alpha \sin \left(d_{1}\right) \tanh \left(\sqrt{-\mu} d_{2}\right)}{\sqrt{-\mu}} t+\zeta_{2}\right)\right) \\
& \times \exp \left(\mathrm{i}\left(d_{1} n+2 \alpha\left(-1+\cos \left(d_{1}\right) \operatorname{sech}^{2}\left(\sqrt{-\mu} d_{2}\right)\right) t+\zeta_{1}\right)\right), \quad \frac{\alpha}{\beta}<0,
\end{aligned}
$$

where $\mu<0, d_{1}, d_{2}, \zeta_{1}$ and $\zeta_{2}$ are arbitrary constants.

Case 2. $\mu>0$. 
In this case, we first derive the expressions $\phi_{n \pm 1}$ in accordance with $(8 b)$ and substitute them along with (32) into equation (31). Then, clearing the denominator and setting the coefficients of $\left(G^{\prime} / G\right)^{l}(0 \leqslant l \leqslant 8)$ to zero, we derive a system of nonlinear algebraic equations for $a_{0}, a_{1}, a_{-1}, d_{1}, d_{2}, c_{1}, c_{2}$ and $\mu$. Solving the system, we get the following solution set:

$\left\{\begin{aligned} c_{1}= & 2 \alpha\left(-1+\cos \left(d_{1}\right) \sec ^{2}\left(\sqrt{\mu} d_{2}\right)\right), \quad c_{2}=-2 \alpha \sin \left(d_{1}\right) \tan \left(\sqrt{\mu} d_{2}\right) / \sqrt{\mu}, \\ & a_{0}=0, \quad a_{-1}=0, \\ a_{1}=\mp \sqrt{-\alpha / \beta} \tan \left(\sqrt{\mu} d_{2}\right) / \sqrt{\mu} & \end{aligned}\right\}$

and the corresponding discrete trigonometric function solution to equation (28) as

$\begin{aligned} u_{n, 4}^{\mp}(t)=\mp & \frac{\sqrt{-\alpha} \tan \left(\sqrt{\mu} d_{2}\right)}{\sqrt{\beta}}\left(\frac{-C_{1} \sin \left(\sqrt{\mu} \xi_{n}\right)+C_{2} \cos \left(\sqrt{\mu} \xi_{n}\right)}{C_{1} \cos \left(\sqrt{\mu} \xi_{n}\right)+C_{2} \sin \left(\sqrt{\mu} \xi_{n}\right)}\right) \\ & \times \exp \left(\mathrm{i}\left(d_{1} n+2 \alpha\left(-1+\cos \left(d_{1}\right) \sec ^{2}\left(\sqrt{\mu} d_{2}\right)\right) t+\zeta_{1}\right)\right), \quad \frac{\alpha}{\beta}<0,\end{aligned}$

where $\xi_{n}=d_{2} n-\frac{2 \alpha \sin \left(d_{1}\right) \tan \left(\sqrt{\mu} d_{2}\right)}{\sqrt{\mu}} t+\zeta_{2}$, and $\mu>0, d_{1}, d_{2}, \zeta_{1}, \zeta_{2}, C_{1}$ and $C_{2}$ remain arbitrary.

As a particular example, if we take ' $C_{1}=0$ and $C_{2} \neq 0$ ' or ' $C_{1} \neq 0$ and $C_{2}=0$ ' in the expression (38) respectively, then we get formal discrete periodic wave solutions to equation (28) as follows:

$$
\begin{gathered}
u_{n, 5}^{\mp}(t)=\mp \frac{\sqrt{-\alpha} \tan \left(\sqrt{\mu} d_{2}\right)}{\sqrt{\beta}} \cot \left(\sqrt{\mu}\left(d_{2} n-\frac{2 \alpha \sin \left(d_{1}\right) \tan \left(\sqrt{\mu} d_{2}\right)}{\sqrt{\mu}} t+\zeta_{2}\right)\right) \\
\times \exp \left(\mathrm{i}\left(d_{1} n+2 \alpha\left(-1+\cos \left(d_{1}\right) \sec ^{2}\left(\sqrt{\mu} d_{2}\right)\right) t+\zeta_{1}\right)\right), \quad \frac{\alpha}{\beta}<0, \\
u_{n, 6}^{\mp}(t)= \pm \frac{\sqrt{-\alpha} \tan \left(\sqrt{\mu} d_{2}\right)}{\sqrt{\beta}} \tan \left(\sqrt{\mu}\left(d_{2} n-\frac{2 \alpha \sin \left(d_{1}\right) \tan \left(\sqrt{\mu} d_{2}\right)}{\sqrt{\mu}} t+\zeta_{2}\right)\right) \\
\times \exp \left(\mathrm{i}\left(d_{1} n+2 \alpha\left(-1+\cos \left(d_{1}\right) \sec ^{2}\left(\sqrt{\mu} d_{2}\right)\right) t+\zeta_{1}\right)\right), \quad \frac{\alpha}{\beta}<0,
\end{gathered}
$$

where $\mu>0, d_{1}, d_{2}, \zeta_{1}$ and $\zeta_{2}$ are arbitrary constants.

Case 3. $\mu=0$.

In this case, we first derive the expressions $\phi_{n \pm 1}$ in accordance with (8c) and substitute them along with (32) into equation (31). Then, clearing the denominator and setting the coefficients of $\left(G^{\prime} / G\right)^{l}(0 \leqslant l \leqslant 7)$ to zero, we derive a system of nonlinear algebraic equations for $a_{0}, a_{1}, a_{-1}, d_{1}, d_{2}, c_{1}$ and $c_{2}$. Solving the system, we get the following solution set:

$\left\{c_{2}=-2 \alpha d_{2} \sin \left(d_{1}\right), c_{1}=2\left(-\alpha+\alpha \cos \left(d_{1}\right)\right), a_{1}=\mp \frac{\sqrt{-\alpha} d_{2}}{\sqrt{\gamma}}, a_{0}=0, a_{-1}=0\right\}$

and the corresponding discrete rational function solution to equation (28) as

$$
\begin{aligned}
u_{n, 7}^{\mp}(t)=\mp \frac{\sqrt{-\alpha} d_{2}}{\sqrt{\beta}}\left(\frac{C_{1}}{C_{1}\left(d_{2} n-2 \alpha d_{2} \sin \left(d_{1}\right) t+\zeta_{2}\right)+C_{2}}\right) & \\
& \times \exp \left(\mathrm{i}\left(d_{1} n+2\left(-\alpha+\alpha \cos \left(d_{1}\right)\right) t+\zeta_{1}\right)\right), \quad \frac{\alpha}{\beta}<0,
\end{aligned}
$$

where $d_{1}, d_{2}, \zeta_{1}, \zeta_{2}, C_{1}$ and $C_{2}$ remain arbitrary.

Note 3. We observe that our solution (35) matches the solution (45) of Huang and Liu [27]. However, our rational solution (42) is not presented in there and derived here for the first time.

Note 4. It is an important fact that one should be aware of the limitations of each of the existing methods. There is no guarantee that they will succeed for a specialized nonlinear 
problem. Any of them can have some advantages and disadvantages. If treated rigorously, the $\left(\mathrm{G}^{\prime} / \mathrm{G}\right)$-expansion method provides exact traveling wave solutions in a neat form from which one can construct solitary and periodic waves, as well as rational ones. One of the pitfalls of the $\left(\mathrm{G}^{\prime} / \mathrm{G}\right)$-expansion method, by assuming the solution of the equation in the polynomial form with many parameters, is that it sometimes leads to inconsistent nonlinear algebraic systems. Another one is that it is entirely algorithmic and involves a large amount of tedious calculations which can become virtually unmanageable if attempted manually. We have encountered no difficulty while working on the relativistic Toda lattice system (9). However, we could not get results for the constraint $\alpha / \beta>0$ while working on the discrete nonlinear Schrödinger equation (28).

\section{Conclusion}

We systematically illustrated the solution procedure of the extended $\left(\mathrm{G}^{\prime} / \mathrm{G}\right)$-expansion method for NDDEs. We obtained a rich variety of discrete traveling wave solutions to a relativistic Toda lattice system and a discrete nonlinear Schrödinger equation. Using a single method, three types of exact solutions are observed: hyperbolic function solutions, trigonometric function solutions and rational function solutions. These obtained solutions with arbitrary parameters may be important to explain some physical phenomena. We would like to point out here that the rational solutions (27) and (42) cannot be obtained by other methods. To the best of our knowledge, these solutions with arbitrary parameters are new; this fact illustrates that our algorithm is effective and more powerful for NDDEs. All solutions are derived here with less algebraic expansion computations with the help of MATHEMATICA. We assured the correctness of our solutions by putting them back into the original equation. More applications of the extended $\left(\mathrm{G}^{\prime} / \mathrm{G}\right)$-expansion method to other types of NDDEs deserve further investigation.

\section{Acknowledgements}

I would like to express my sincere thanks to the referees for their valuable suggestions and comments.

\section{References}

[1] Fermi E, Pasta J and Ulam S 1965 Collected Papers of Enrico Fermi II (Chicago, IL: University of Chicago Press)

[2] Toda M 1989 Theory of Nonlinear Lattices (Berlin: Springer)

[3] Wadati M 1976 Transformation theories for nonlinear discrete systems Prog. Suppl. Theor. Phys. 59 36-63

[4] Ohta Y and Hirota R 1991 A discrete KdV equation and its Casorati determinant solution J. Phys. Soc. Japan 602095

[5] Ablowitz M J and Ladik J 1975 Nonlinear differential-difference equations J. Math. Phys. 16 598-603

[6] Wang M, Li X and Zhang J 2008 The $\left(\mathrm{G}^{\prime} / \mathrm{G}\right)$-expansion method and traveling wave solutions of nonlinear evolution equations in mathematical physics Phys. Lett. A 372 417-23

[7] Wang M, Zhang J and Li X 2008 Application of the $\left(\mathrm{G}^{\prime} / \mathrm{G}\right)$-expansion to travelling wave solutions of the Broer-Kaup and the approximate long water wave equations Appl. Math. Comput. 206 321-6

[8] Zayed E M E and Gepreel K A 2009 Some applications of the ( $\left.\mathrm{G}^{\prime} / \mathrm{G}\right)$-expansion method to non-linear partial differential equations Appl. Math. Comput. 212 1-13

[9] Ling-Xiao L and Ming-Liang W 2009 The $\left(\mathrm{G}^{\prime} / \mathrm{G}\right)$-expansion method and travelling wave solutions for a higher-order nonlinear Schrödinger equation Appl. Math. Comput. 208 440-5

[10] Aslan İ and Öziş T 2009 Analytic study on two nonlinear evolution equations by using the (G' $/ G)$-expansion method Appl. Math. Comput. 209 425-9 
[11] Aslan İ and Öziş T 2009 On the validity and reliability of the $\left(\mathrm{G}^{\prime} / \mathrm{G}\right)$-expansion method by using higher-order nonlinear equations Appl. Math. Comput. 211 531-6

[12] Öziş T and Aslan İ 2009 Symbolic computation and construction of new exact traveling wave solutions to Fitzhugh-Nagumo and Klein-Gordon equations Z. Naturforsch. 64a 15-20

[13] Öziş T and Aslan İ 2009 Symbolic computations and exact and explicit solutions of some nonlinear evolution equations in mathematical physics Commun. Theor. Phys. 51 577-80

[14] Aslan İ 2009 Exact and explicit solutions to some nonlinear evolution equations by utilizing the $\left(\mathrm{G}^{\prime} / \mathrm{G}\right)$ expansion method Appl. Math. Comput. 215 857-63

[15] Zhang J, Wei X and Lu Y 2008 A generalized ( $\left.\mathrm{G}^{\prime} / \mathrm{G}\right)$-expansion method and its applications Phys. Lett. A $\mathbf{3 7 2} 3653-8$

[16] Zhang S, Wang W and Tong J 2009 A generalized (G'/G)-expansion method and its application to the (2+1)dimensional Broer-Kaup equations Appl. Math. Comput. 209 399-404

[17] Zhang S, Tong J L and Wang W 2008 A generalized $\left(\mathrm{G}^{\prime} / \mathrm{G}\right)$-expansion method for the mKdV equation with variable coefficients Phys. Lett. A 372 2254-7

[18] Yu-Bin Z and Chao L 2009 Application of modified $\left(\mathrm{G}^{\prime} / \mathrm{G}\right)$-expansion method to traveling wave solutions for Whitham Broer Kaup-Like equations Commun. Theor. Phys. 51 664-70

[19] Zhang H 2009 New application of the $\left(\mathrm{G}^{\prime} / \mathrm{G}\right)$-expansion method Commun. Nonlinear Sci. Numer. Simul. 14 3220-5

[20] Zhang S, Dong L, Ba J and Sun Y 2009 The $\left(\mathrm{G}^{\prime} / \mathrm{G}\right)$-expansion method for nonlinear differential-difference equations Phys. Lett. A 373 905-10

[21] Aslan İ 2009 Discrete exact solutions to some nonlinear differential-difference equations via the $\left(\mathrm{G}^{\prime} / \mathrm{G}\right)$ expansion method Appl. Math. Comput. 215 3140-7

[22] Aslan I 2010 Application of the $\left(\mathrm{G}^{\prime} / \mathrm{G}\right)$-expansion method to Kawahara type equations using symbolic computation Appl. Math. Comput. 216 2360-5

[23] Aslan İ 2010 The Ablowitz-Ladik lattice system by means of the extended (G'/G)-expansion method Appl. Math. Comput. 216 2778-82

[24] Aslan İ 2010 A note on the ( $\left.\mathrm{G}^{\prime} / \mathrm{G}\right)$-expansion method again Appl. Math. Comput. 217 937-8

[25] Baldwin D, Goktas U and Hereman W 2004 Symbolic computation of hyperbolic tangent solutions for nonlinear differential-difference equations Comput. Phys. Commun. 162 203-17

[26] Ablowitz M J and Ladik J F 1976 A nonlinear difference scheme and inverse scattering Stud. Appl. Math. 55 213-29

[27] Huang W and Liu Y 2008 Doubly periodic wave solutions and soliton solutions of Ablowitz-Ladik lattice system Int. J. Theor. Phys. $47338-49$ 